\title{
Drug usage pattern of antimicrobials in elderly hypertensive, diabetic in-patients with or without impaired renal function
}

\author{
Prasan R. Bhandari*, Apeksha Bhandary
}

Department of Pharmacology, SDM College of Medical Sciences and Hospital, Dharwad, Karnataka, India

Received: 18 July 2018 Accepted: 29 August 2018

*Correspondence to: Dr. Prasan R. Bhandari, Email: prasangeeta2012@ gmail.com

Copyright: () the author(s), publisher and licensee Medip Academy. This is an openaccess article distributed under the terms of the Creative Commons Attribution NonCommercial License, which permits unrestricted noncommercial use, distribution, and reproduction in any medium, provided the original work is properly cited.

\begin{abstract}
Background: Hypertension (HTN) and diabetes mellitus (DM) are the primary contributors to renovascular mortality and morbidity including chronic kidney diseases. Additionally, these patients are in frequent need of an antimicrobial agent. Drug utilisation studies (DUS) are prospective tools in the assessment of health care systems. The objective of the present study was to analyse the prescription pattern of anti-microbials in elderly hypertensive diabetic in-patients with or without renal impairment in a tertiary hospital

Methods: The study population comprised of 165 hypertensive diabetic inpatients at Shri Dharmasthala Manjunatheshwara (SDM) Hospital. Questionnaire based evaluation was carried out and prescriptions of patient with HTN and DM at and above the age of 60 years irrespective of gender were included.

Results: Among anti-microbial agents, other $\beta$ lactam antibacterial were the most commonly prescribed class of drugs $(40.85 \%)$ which was similar in patients with impaired renal function $(21.8 \%)$.

Conclusions: There was a significant increase in the number of anti-microbial agents and other drugs in the patients with impaired renal function when compared to patients with normal renal function $(\mathrm{p}<0.05)$.
\end{abstract}

Keywords: Antimicrobial, Drug utilization, Renal impairment

\section{INTRODUCTION}

Chronic kidney disease (CKD) is characterized by multiple disorders affecting the morphology and function of kidneys. ${ }^{1}$ It is estimated on the basis of a decrease in the number of nephrons, which ultimately decreases the glomerular filtration rate (GFR) for a period more than 3 months. ${ }^{2}$ Hypertension and diabetes, recurrent infections along with an inappropriate prescription of drugs are the leading factors resulting in the increasing incidence of CKD. ${ }^{3,4}$ CKD accounts for 850,000 deaths worldwide as reported by World Health Organization. ${ }^{5}$

Pharmacokinetics in renal compromised patients is altered and often results in drug accumulation and toxicity. ${ }^{6}$ Diabetes, hypertension, and renovascular diseases are the common causes of CKD in developing countries, whereas in the tropical countries, infection induced glomerular disease contributes the most to $\mathrm{CKD} .^{3,7}$ Hence, the utilization pattern, marketing and distribution of drugs widely varies with time, geographical distribution, health economics, and sociomedical parameters. ${ }^{8}$

Due to diverse co-morbid conditions and complications, physicians have to use multiple drugs in the management of CKD, which, on the contrary, results to drug interaction and suboptimal action. CKD patients are more susceptible to infections and are likely to be prescribed with antimicrobial agents. The dosing of all drugs, including antibiotics should be optimized and monitored so as to prevent adverse drug reactions, avoid further renal injury and to facilitate treatment outcomes. ${ }^{9-11}$

Antimicrobials may well be required in CKD patients for reasons such as urinary or respiratory tract infections. However, since many antibiotics are renally eliminated 
and some may be nephrotoxic in nature, careful antibiotic drug and dose selection are necessary. The lack of a clear indication for antibiotic therapy in $82.05 \%$ cases is, therefore, a matter of concern.

Drug utilisation studies carries an important role in clinical scenarios as it is the base for generating amendments in the drug dispensing guidelines at local and national levels. The ensuing objective of such study is to support rational drug use. Additionally, as it assists in formulating policies to utilize health resources in the most effective method, it is essentially necessary in a developing economy like India where health care burden is endured by the patients.

The reference standard for drug utilisation is WHO ATC/DDD (Anatomical Therapeutic Chemical/Defined daily dose) methodology. ${ }^{12}$ Defined daily dose (DDD) is defined by the WHO Collaborating Centre for Drug Statistics and Methodology as the expected average maintenance adult dose per day for its main indication for each drug and route of administration. The DDD thus is an international unit assisting in international or regional comparisons. However, DDD does not fundamentally disclose the recommended or prescribed daily dose (PDD). Nonetheless, a number of studies have described inconsistencies between DDD and PDD for different groups of drugs. ${ }^{13-16}$ Hence a drug utilization study was conducted to evaluate the drug utilization pattern of antimicrobial medicines during hospital stay and at the time of discharge, cost of antidiabetic drugs and defined daily dose (DDD)/100 bed-days during hospital stay in our setup.

DUS can identify the frequent prescribing errors, their causes, the deviation from the guidelines, and the cost effectiveness. In the present study we intend to:

- Analyse the drug usage pattern of anti-microbials in elderly hypertensive diabetic in-patients in a tertiary hospital.

- Analyse drug usage pattern of anti-microbials in elderly hypertensive, diabetic in-patients with renal impairment in a tertiary hospital.

\section{METHODS}

\section{Study design}

It was a prospective, observational study carried out at medicine wards in SDM College of Medical Sciences and Hospital, Karnataka. The study protocol was approved by the Institutional ethics committee (IEC). All the patients were explained clearly about the nature and purpose of the study in their own language and consent was taken.

\section{Inclusion criteria}

- Patients of either sex at and above 60 years admitted in the medicine wards diagnosed with HTN and DM and were on treatment with anti-diabetics,
- Patients whose renal profile (Serum creatinine) is available after their admission to the hospital.

\section{Exclusion criteria}

- Patients less than 60 years of age,

- Patients diagnosed with HTN or DM but not both,

- Patients whose renal profile data was not available,

- Patient/ relative who were not willing to give their consent or were unable to give consent,

- No sufficient data (age, registration number) were available,

- Patients having emergency/life threatening medical/ surgical conditions.

\section{Sample size}

A total of 165 hypertensive and diabetic patients clinically diagnosed as per JNC 7 and American Diabetes Association (ADA) at SDM Medicine wards were enrolled.

\section{Participants and data collection}

A questionnaire-based study was conducted at the medicine wards. Prescriptions of clinically diagnosed HTN and DM patients at and above the age of 60 years admitted in the medicine wards were included. The questionnaire consisted patient's demographics like age, gender, marital status, religion and registration number. Patient's diagnosis was made as per JNC 7 and ADA 2015. Renal function tests were obtained and creatinine clearance $(\mathrm{Cr} . \mathrm{Cl})$ calculated using Cockroft-Gault equation. BP recording and BS levels, (Random blood sugar (RBS), fasting blood sugar (FBS), and glycosylated haemoglobin $(\mathrm{HbA} 1 \mathrm{c})$, if done, then the values were noted.

\section{Statistical analysis}

Descriptive statistics was applied. Data was analysed by proportion and percentages and comparison done using chi-square test using Statistical Package for the Social Sciences (SPPS) software version 24.

\section{RESULTS}

In the study population majority $88(53.33 \%)$ of the patients had impaired renal function and $77(46.67 \%)$ of patients had normal renal function (Figure 1). A total of 142 antimicrobial agents excluding FDCs were prescribed for 165 patients. Among them ATC class J01D (other $\beta$ lactam antibacterials) was most commonly used $40.85 \%$ $(\mathrm{n}=58)$ of which ceftriaxone was used more frequently (35.21\%), followed by class J01F (macrolides, lincosamides and streptogramins) (13.38\%). Among patients with impaired renal function too ceftriaxone was the commonest drug prescribed $(17.6 \%)$ (Table 1$)$. 
Table 1: Antimicrobial agents prescribed to the study population.

\begin{tabular}{|c|c|c|c|c|c|c|c|c|c|c|c|c|c|}
\hline \multicolumn{7}{|c|}{ Class/ATC classification } & \multicolumn{7}{|l|}{ Generic name } \\
\hline \multirow{3}{*}{ ATC } & \multirow{2}{*}{\multicolumn{2}{|c|}{ Total }} & \multicolumn{4}{|c|}{ Impaired renal function } & \multirow[b]{3}{*}{ Drug name } & \multirow{2}{*}{\multicolumn{2}{|c|}{ Total }} & \multicolumn{4}{|c|}{$\begin{array}{l}\text { Impaired renal } \\
\text { function }\end{array}$} \\
\hline & & & \multicolumn{2}{|l|}{ Yes } & \multicolumn{2}{|l|}{ No } & & & & \multicolumn{2}{|c|}{ Yes } & \multicolumn{2}{|l|}{ No } \\
\hline & $\mathbf{n}$ & $\%$ & $\mathbf{n}$ & $\%$ & $\mathbf{n}$ & $\%$ & & n & $\%$ & $\mathbf{n}$ & $\%$ & $\mathbf{n}$ & $\%$ \\
\hline J01A & 8 & 5.63 & 6 & 4.2 & 2 & 1.4 & J01AA02 - Doxycycline & 8 & 5.63 & 6 & 4.2 & 2 & 1.4 \\
\hline J01C & 1 & 0.70 & 1 & 0.7 & 0 & 0.0 & J01CA01 - Ampicillin & 1 & 0.7 & 1 & 0.7 & 0 & 0.0 \\
\hline \multirow{5}{*}{ J01D } & \multirow{5}{*}{58} & \multirow{5}{*}{40.85} & \multirow{5}{*}{31} & \multirow{5}{*}{21.8} & \multirow{5}{*}{27} & \multirow{5}{*}{19.0} & J01DC02 - Cefuroxime & 3 & 2.11 & 2 & 1.4 & 1 & 0.7 \\
\hline & & & & & & & J01DD04 - Ceftriaxone & 50 & 35.21 & 25 & 17.6 & 25 & 17.6 \\
\hline & & & & & & & J01DE01 - Cefepime & 1 & 0.7 & 1 & 0.7 & 0 & 0.0 \\
\hline & & & & & & & J01DH02 - Meropenem & 3 & 2.11 & 2 & 1.4 & 1 & 0.7 \\
\hline & & & & & & & J01DI03 - Faropenem & 1 & 0.7 & 1 & 0.7 & 0 & 0.0 \\
\hline \multirow{2}{*}{ J01F } & \multirow{2}{*}{19} & \multirow{2}{*}{13.38} & \multirow{2}{*}{10} & \multirow{2}{*}{7.0} & \multirow{2}{*}{9} & \multirow{2}{*}{6.3} & J01FA09 - Clarithromycin & 9 & 6.34 & 5 & 3.5 & 4 & 2.8 \\
\hline & & & & & & & J01FA10 - Azithromycin & 10 & 7.04 & 5 & 3.5 & 5 & 3.5 \\
\hline J01G & 1 & 0.70 & 0 & 0.0 & 1 & 0.7 & J01GB06 - Amikacin & 1 & 0.7 & 0 & 0.0 & 1 & 0.7 \\
\hline \multirow{2}{*}{ J01M } & \multirow{2}{*}{10} & 704 & 6 & 42 & 4 & 28 & J01MA12 - Levofloxacin & 3 & 2.11 & 2 & 1.4 & 1 & 0.7 \\
\hline & & 1.04 & 0 & 4.2 & 4 & 2.8 & J01MA14 - Moxifloxacin & 8 & 5.63 & 5 & 3.5 & 3 & 2.1 \\
\hline $\mathrm{J} 01 \mathrm{X}$ & 18 & 12.68 & 11 & 7.7 & 7 & 4.9 & J01XA02 - Teicoplanin & 6 & 4.23 & 5 & 3.5 & 1 & 0.7 \\
\hline & & & & & & & J01XE01 - Nitrofurantoin & 3 & 2.11 & 2 & 1.4 & 1 & 0.7 \\
\hline & & & & & & & J01XX08 - Linezolid & 9 & 6.34 & 4 & 2.8 & 5 & 3.5 \\
\hline J02A & 2 & 1.41 & 0 & 0.0 & 2 & 0.0 & J02AC01 - Fluconazole & 2 & 1.41 & 0 & 0.0 & 2 & 1.4 \\
\hline P01A & 18 & 12.68 & 12 & 8.5 & 6 & 4.2 & P01AB01 -Metronidazole & 6 & 4.23 & 1 & 0.7 & 5 & 3.5 \\
\hline & & & & & & & P01AB03 - Ornidazole & 11 & 7.75 & 10 & 7.0 & 1 & 0.7 \\
\hline P01B & 1 & 0.70 & 0 & 0.0 & 1 & 0.7 & P01BE03 - Artesunate & 1 & 0.7 & 0 & 0.0 & 1 & 0.7 \\
\hline $\mathrm{P} 02 \mathrm{C}$ & 6 & 4.23 & 3 & 2.1 & 3 & 2.1 & P02CA03 - Albendazole & 6 & 4.23 & 3 & 2.1 & 3 & 2.1 \\
\hline Total & 142 & 100.0 & 80 & 56.3 & 62 & 43.7 & Total & 142 & 100.0 & 80 & 56.3 & 62 & 43.7 \\
\hline
\end{tabular}

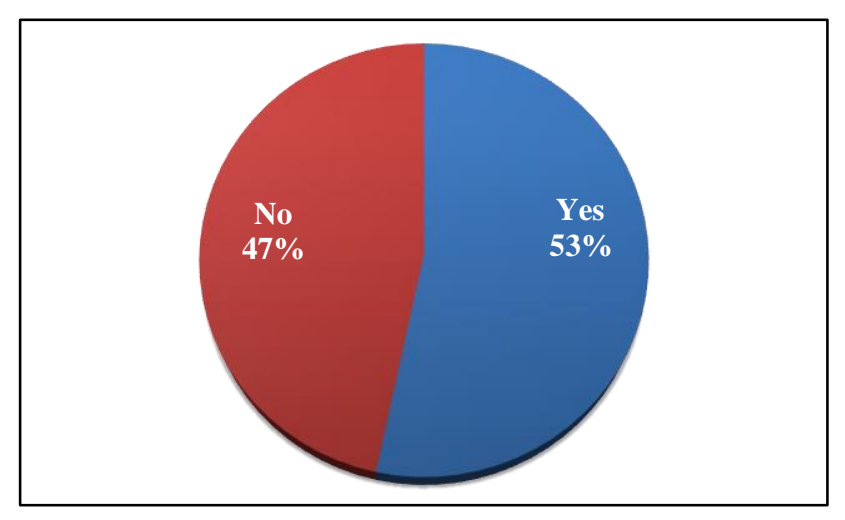

Figure 1: Impaired renal function in the study participants.

The average PDD/DDD for the most commonly prescribed drugs ceftriaxone and ornidazole was 1 and 1.28 respectively in our study population. PDD/DDD ratio ranged between (0.30-2.25), least being for cefepime (0.26) and maximum for doxycycline (2.25). The range of PDD/DDD for drugs with impaired renal function was (0.28-2.30). For faropenem no DDD is defined when the study was conducted (Table 2).

Among the 142 anti-microbial agents prescribed majority of drugs were BD dosing 95 (66.90\%), followed by OD dosing 29 (20.42\%). IV route of administration was the most commonly used route prescribed for antimicrobial agents, $113(79.58 \%)$, followed by oral route $29(20.42 \%)$ (Table 3).

A total of 88 antimicrobial FDCs were prescribed for 165 patients. Among them ATC class J01CR (Combination of penicillins inclusive of $\beta$ lactamase inhibitors) was most commonly used $47.7 \%$ (42) of which piperacillin + tazobactum was used more frequently $(38.6 \%)$, followed by class J01DD ( $3^{\text {rd }}$ generation cephalosporins) $(37.5 \%)$. Among patients with impaired renal function too piperacillin + tazobactum was the commonest FDCs prescribed (29.5\%) (Table 4).

The average PDD/DDD for the most commonly prescribed FDC drugs piperacillin + tazobactum and cefoperazone + tazobactum was 0.63 and 0.85 respectively in our study population. PDD/DDD ratio ranged between $(0.37-0.71)$, least being for cefoperazone + sulbactum $(0.37)$ and maximum for amoxicillin + clavulanic acid (0.71). The range of PDD/DDD for drugs with impaired renal function was $(0.16-0.75)$ (Table 5).

Among the 88 antimicrobial FDCs prescribed majority of drugs were BD dosing $69(78.41 \%)$, followed by TID dosing $16(18.18 \%)$. IV route of administration was the 
most commonly prescribed route of administration $(94.3 \%)$ for antimicrobial FDCs (Table 6).

Table 2: Average PDD/DDD of antimicrobial agents in the study population.

\begin{tabular}{|c|c|c|c|}
\hline \multicolumn{4}{|l|}{ Avg. PDD/DDD } \\
\hline & \multirow{2}{*}{ Total } & \multicolumn{2}{|c|}{ Impaired renal function } \\
\hline & & Yes & No \\
\hline Albendazole & 1.00 & 1.00 & 1.00 \\
\hline Amikacin & 1.00 & 0 & 1.00 \\
\hline Ampicillin & 1.00 & 1.00 & 0.00 \\
\hline Artesunate & 0.43 & 0 & 0.43 \\
\hline Azithromycin & 1.47 & 1.40 & 1.53 \\
\hline Cefepime & 0.30 & 0.30 & 0.00 \\
\hline Ceftriaxone & 1.00 & 1.00 & 1.00 \\
\hline Cefuroxime & 0.67 & 0.80 & 0.50 \\
\hline Clarithromycin & 1.67 & 1.80 & 1.50 \\
\hline Doxycycline & 2.25 & 2.30 & 2.00 \\
\hline Faropenem & - & - & - \\
\hline Fluconazole & 0.88 & 0.00 & 0.87 \\
\hline Levofloxacin & 1.00 & 1.00 & 1.00 \\
\hline Linezolid & 1.00 & 1.00 & 1.00 \\
\hline Meropenem & 1.17 & 1.00 & 1.50 \\
\hline Metronidazole & 0.94 & 1.00 & 0.93 \\
\hline Moxifloxacin & 1.13 & 1.20 & 1.00 \\
\hline Nitrofurantoin & 1.33 & 1.50 & 1.00 \\
\hline Ornidazole & 1.28 & 1.20 & 1.50 \\
\hline Teicoplanin & 0.58 & 0.60 & 0.50 \\
\hline
\end{tabular}

Out of 165 patients, $42.42 \%$ (70) of patients C/S tests was done and $57.58 \%$ (95) of patients $\mathrm{C} / \mathrm{S}$ was not done. Out of $70 \mathrm{C} / \mathrm{S}$ reports in the study participants, most common (17.14\%) organism found was Escherichia coli, followed by enterococcus species $(8.57 \%)$. No growth in the C/S was seen in $41.43 \%$ (29) of patients (Figure 2 and 3).

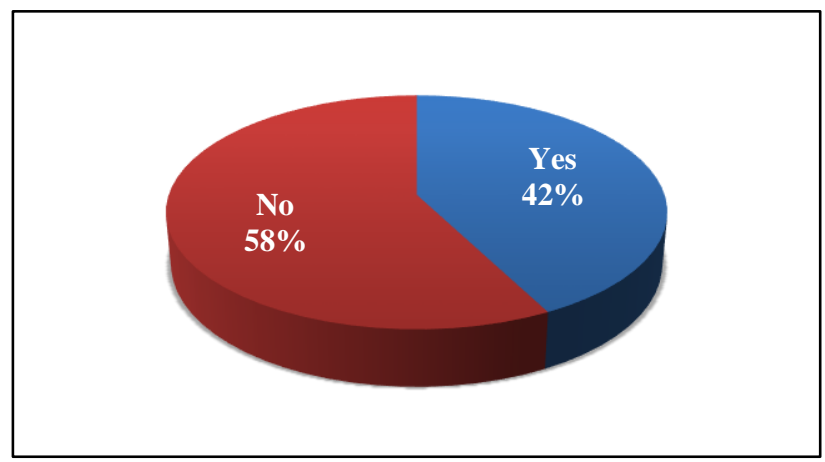

Figure 2: Culture and sensitivity done in the study participants.

Table 3: Frequency and route of administration of antimicrobial agents in the study population.

\begin{tabular}{|llllll|}
\hline Frequency & $\mathbf{n}$ & $\%$ & Route & n & \% \\
\cline { 1 - 3 } OD & 29 & 20.42 & IV & 113 & 79.58 \\
\hline BD & 95 & 66.90 & & & \\
TID & 10 & 7.04 & Oral & 29 & 20.42 \\
\cline { 1 - 3 } STAT & 8 & 5.63 & & & \\
\hline Total & 142 & 100.00 & Total & 142 & 100.0 \\
\hline
\end{tabular}

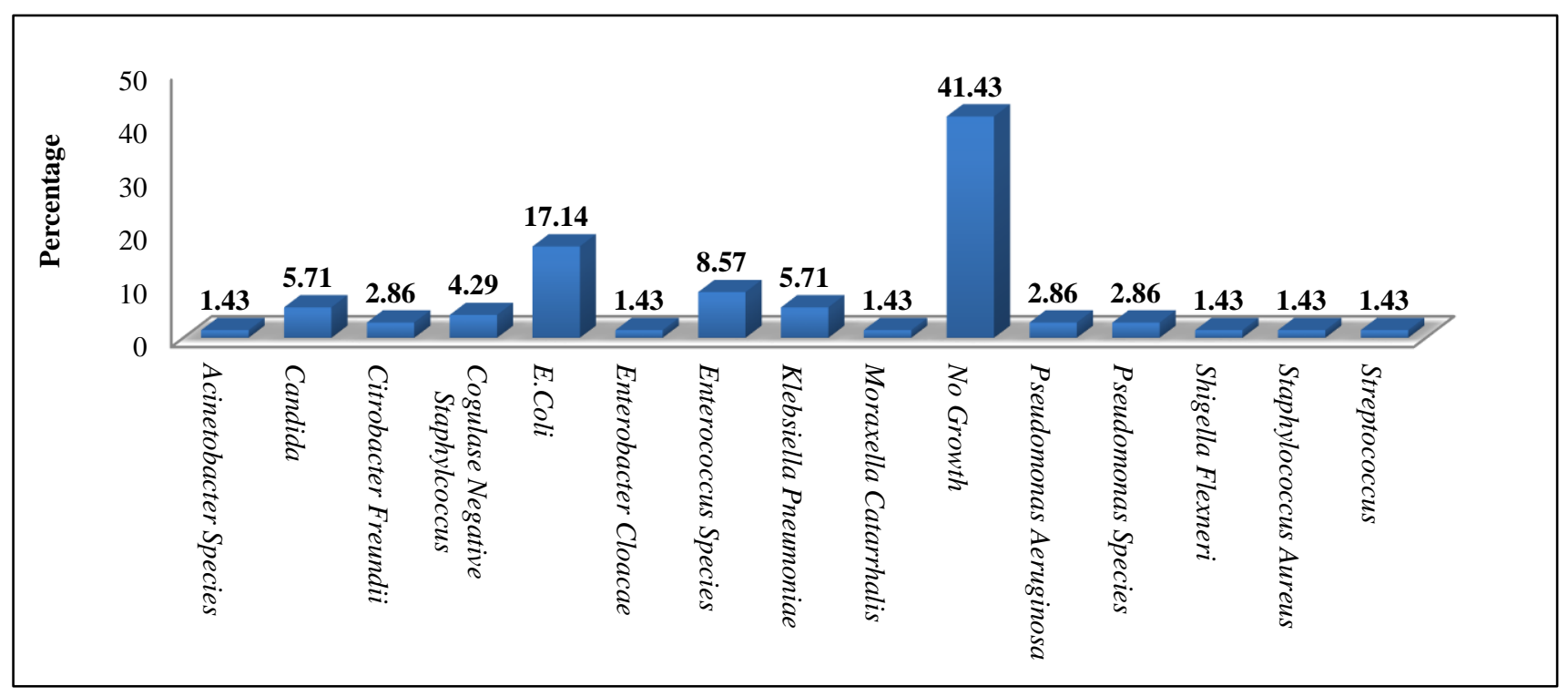

Figure 3: Microorganisms found in the C/S tests in the study population.

Out of $70 \mathrm{C} / \mathrm{S}$ reports in the study participants, a total of 350 antimicrobial agents were found to be resistant.
Maximum, $26(7.43 \%)$ antimicrobial resistance was seen for the drug ciprofloxacin, followed by ampicillin $5.43 \%$ 
(19), cefuroxime $5.43 \%$ (19), co-trimoxazole $5.43 \%$ (19), gentamycin $5.43 \%$ (19), ceftriaxone $4.86 \%$ (17), nalidixic acid $4.86 \%$ (17), amoxicillin $4.57 \%$ (16), cefepime $4.57 \%$ (16), cefotaxime $4.29 \%$ (15), cefuroxime axetil $4.29 \%$
(15), amoxicillin + clavulanic acid 3.14\% ( $\mathrm{n}=11)$, clindamycin $2.29 \% \quad(8)$, erythromycin $2.00 \% \quad(7)$, levofloxacin $2.00 \%$ (7), tetracycline $2.00 \%$ (7).

Table 4: Antimicrobial FDCs prescribed for the study population.

\begin{tabular}{|c|c|c|c|c|c|c|c|c|c|c|c|c|c|}
\hline \multicolumn{7}{|c|}{ Class/ATC classification } & \multicolumn{7}{|l|}{ Generic name } \\
\hline \multirow{3}{*}{ ATC code } & \multirow{2}{*}{\multicolumn{2}{|c|}{ Total }} & \multicolumn{4}{|c|}{ Impaired renal function } & \multirow[b]{3}{*}{ Drug name } & \multirow{2}{*}{\multicolumn{2}{|c|}{ Total }} & \multicolumn{4}{|c|}{ Impaired renal function } \\
\hline & & & \multicolumn{2}{|c|}{ Yes } & \multicolumn{2}{|c|}{ No } & & & & \multicolumn{2}{|c|}{ Yes } & \multicolumn{2}{|c|}{ No } \\
\hline & $\mathbf{n}$ & $\%$ & $\mathbf{n}$ & $\%$ & $\mathbf{n}$ & $\%$ & & $\mathbf{n}$ & $\%$ & $\mathbf{n}$ & $\%$ & $\mathbf{n}$ & $\%$ \\
\hline A02BD & 1 & 1.1 & 1 & 1.1 & 0 & 0.0 & $\begin{array}{l}\text { A02BD06-Amoxicillin+ } \\
\text { Clarithromycin+ } \\
\text { Esomeprazole }\end{array}$ & 1 & 1.1 & 1 & 1.1 & 0 & 0.0 \\
\hline \multirow{2}{*}{ J01CR } & \multirow{2}{*}{42} & \multirow{2}{*}{47.7} & \multirow{2}{*}{30} & \multirow{2}{*}{34.1} & \multirow{2}{*}{12} & \multirow{2}{*}{13.6} & $\begin{array}{l}\text { J01CR02- } \\
\text { Amoxiciliin+Clavulanic Acid }\end{array}$ & 8 & 9.1 & 4 & 4.5 & 4 & 4.5 \\
\hline & & & & & & & $\begin{array}{l}\text { J01CR05- } \\
\text { Piperacillin+Tazobactum }\end{array}$ & 34 & 38.6 & 26 & 29.5 & 8 & 9.1 \\
\hline \multirow{3}{*}{ J01DD } & \multirow{3}{*}{33} & \multirow{3}{*}{37.5} & \multirow{3}{*}{25} & \multirow{3}{*}{28.4} & \multirow{3}{*}{8} & \multirow{3}{*}{9.1} & $\begin{array}{l}\text { J01DD54- } \\
\text { Ceftriaxone+Tazobactum }\end{array}$ & 12 & 13.6 & 11 & 12.5 & 1 & 1.1 \\
\hline & & & & & & & $\begin{array}{l}\text { J01DD62- } \\
\text { Cefoperazone+Sulbactum }\end{array}$ & 4 & 4.5 & 3 & 3.4 & 1 & 1.1 \\
\hline & & & & & & & $\begin{array}{l}\text { J01DD62- } \\
\text { Cefoperazone+Tazobactum }\end{array}$ & 17 & 19.3 & 11 & 12.5 & 6 & 6.8 \\
\hline J01DH & 4 & 4.5 & 4 & 4.5 & 0 & 0.0 & $\begin{array}{l}\text { J01DH55- } \\
\text { Imipenem+Cilastatin }\end{array}$ & 4 & 4.5 & 4 & 4.5 & 0 & 0.0 \\
\hline \multirow{2}{*}{ J01R } & \multirow{2}{*}{5} & \multirow{2}{*}{5.7} & \multirow{2}{*}{2} & \multirow{2}{*}{2.3} & \multirow{2}{*}{3} & \multirow{2}{*}{3.4} & J01R-Cefepime+Tazobactum & 4 & 4.5 & 1 & 1.1 & 3 & 3.4 \\
\hline & & & & & & & J01R-Ornidazole+ Cefixime & 1 & 1.1 & 1 & 1.1 & 0 & 0.0 \\
\hline J01RA & 2 & 2.3 & 0 & 0.0 & 2 & 2.3 & $\begin{array}{l}\text { J01RA09-Ornidazole+ } \\
\text { Ofloxacin }\end{array}$ & 2 & 2.3 & 0 & 0.0 & 2 & 2.3 \\
\hline J04AM & 1 & 1.1 & 0 & 0.0 & 1 & 1.1 & $\begin{array}{l}\text { J04AM06- } \\
\text { Isoniazid+Rifampicin+ } \\
\text { Pyrizinamide+Ethambutol }\end{array}$ & 1 & 1.1 & 0 & 0.0 & 1 & 1.1 \\
\hline Total & 88 & 100 & 62 & 70.5 & 26 & 29.5 & Total & 88 & 100 & 62 & 70.5 & 26 & 29.5 \\
\hline
\end{tabular}

Table 5: Average PDD/DDD of antimicrobial FDC in the study population.

\begin{tabular}{|c|c|c|c|}
\hline \multirow[t]{2}{*}{ Drug name } & \multirow[t]{2}{*}{ Total } & \multicolumn{2}{|c|}{$\begin{array}{l}\text { Impaired } \\
\text { renal } \\
\text { function }\end{array}$} \\
\hline & & Yes & No \\
\hline $\begin{array}{l}\text { Amoxicillin+Clarithromycin+ } \\
\text { Esomeprazole }\end{array}$ & - & - & - \\
\hline Amoxicillin+Clavulanic Acid & 0.71 & 0.75 & 0.66 \\
\hline Cefepime+Tazobactum & - & - & - \\
\hline Cefoperazone+Sulbactum & 0.37 & 0.16 & 0.5 \\
\hline Cefoperazone+Tazobactum & 0.85 & 0.82 & 0.92 \\
\hline Ceftriaxone+Tazobactum & - & - & - \\
\hline Imipenem+Cilastatin & 0.56 & 0.56 & 0.00 \\
\hline $\begin{array}{l}\text { Isoniazid+Rifampicin+ } \\
\text { Pyrizinamide+Ethambutol }\end{array}$ & - & - & - \\
\hline Ornidazole+Cefixime & - & - & - \\
\hline Ornidazole+Ofloxacin & - & - & - \\
\hline Piperacillin+Tazobactum & 0.63 & 0.60 & 0.71 \\
\hline
\end{tabular}

Table 6: Frequency and route of administration for antimicrobial FDCs in the study population.

\begin{tabular}{|c|c|c|c|c|c|}
\hline Frequency & $\mathbf{n}$ & $\%$ & Route & $\mathbf{N}$ & $\%$ \\
\hline OD & 2 & 2.27 & \multirow{2}{*}{ IV } & \multirow{2}{*}{83} & \multirow{2}{*}{94.3} \\
\hline $\mathrm{BD}$ & 69 & 78.41 & & & \\
\hline TID & 16 & 18.18 & \multirow{2}{*}{ Oral } & \multirow{2}{*}{5} & \multirow{2}{*}{5.7} \\
\hline QID & 1 & 1.14 & & & \\
\hline Total & 88 & 100.0 & Total & 88 & 100.0 \\
\hline
\end{tabular}

\section{DISCUSSION}

Among 230 antimicrobial agents prescribed for 165 patients, 88 were FDCs and 142 single drugs. In patients with impaired renal function a total of 142 antimicrobial agents were prescribed. There was a significant difference in the no. of drugs used in the patients with impaired renal function and in patients with normal renal function, ( $p$ $<0.05)$. Out of 142 drugs, the most common, $40.85 \%$ $(n=58)$ class of antimicrobial agents prescribed were other $\beta$ lactam antibacterials, followed by macrolides, 
linocosamides and streptogramins, $13.38 \% \quad(n=19)$. Ceftriaxone was the most frequently $35.21 \% \quad(n=50)$ prescribed antimicrobial agent, followed by ornidazole $7.75 \%(n=11)$, azithromycin $7.04 \%(n=10)$. This was in contrast to a study on patients with DM conducted by Ramachandran $\mathrm{G}$ et al, where fluroquinolones was most commonly prescribed $(48 \%)$, followed by penicillins and cephalosporins $(11 \%)$ each. ${ }^{17}$

In patients with impaired renal function $56.3 \% \quad(n=80)$ antimicrobial agents were used with $\beta$ lactam antibacterials being the most frequently prescribed, $21.8 \% \quad(n=31)$, followed by anti-amoebic drugs $8.5 \%(n=12)$. Ceftriaxone was the most common drug prescribed in patients with impaired renal function, $17.6 \% \quad(n=25)$ followed by ornidazole, $7 \%(n=10)$ when compared to patients with normal renal function where ceftriaxone was prescribed, $17.6 \%(n=25)$ and ornidazole only once $(0.7 \%)$.

PDD/DDD ratio ranged between $(0.30-2.25)$, least being for cefepime (0.26) and maximum for doxycycline (2.25). The range of PDD/DDD for drugs with impaired renal function was $(0.28-2.30)$.

A total of 88 FDCs were prescribed, among which penicillins in combination with $\beta$ lactamase inhibitors were frequently prescribed, $47.7 \% \quad(n=42)$. Out of this piperacillin + tazobactum combination was prescribed in majority, $38.6 \%$ (34). Next in line was combination of cephalosporins, $37.5 \%(n=33)$, out of this cefoperazone + tazobactum was frequently prescribed (29.5\%). Among patients with impaired renal function 62 (70.5\%) FDCs were prescribed, which were more in number to that of patients with normal renal function to whom merely $29.5 \%$ $(n=26)$ were prescribed. In a study conducted by Santra et al, in patients with CKD azithromycin and levofloxacin were the two drugs most frequently prescribed oral antimicrobial agents used. Cefoperazone, piperacillin + tazobactum, on the other hand, were the most utilized parenteral antibiotics which was similar to present study. ${ }^{18}$

A total of 230 antimicrobial agents were prescribed among which 88 were FDCs. Other $\beta$ lactam antibacterials were the most common class of drug prescribed and combination of penicillins with $\beta$ lactamase inhibitors were the most common FDCs prescribed in patients with or without impaired renal function.

As per the WHO indicators, the average no. of drugs per patient was $9.64 \pm 3.123$ and with patients with impaired renal function tests was $10.05 \pm 3.081$, with the least being 4/patient and maximum upto 20/patient. This is at par compared to study by Abraham et al, in elderly where the average no. of drugs is 9.09 , is less compared to study by Bajait et al, in patients with CKD where the average is 9.47, and is more when compared to study by Santra et al, in patients with CKD, where the average is $8.1 \pm 3.2 .^{17,19,20}$ The practice of polypharmacy is a common finding in similar studies in CKD patients with average number of drugs per prescription varying from 8 to 12.3 . In this study only, the prescribed medicines were considered but it is well-known that over the counter use of medicines is common in India. This further increases the chances of drug interactions and adverse reactions. ${ }^{21}$

Percentage of drugs prescribed by generic names is $6 \%$ $(n=92)$ which is much lower to the study conducted by Jhaveri et al, where $48.79 \%$ of drugs were prescribed by generic names. ${ }^{22}$

Percentage of encounters with an antibiotic prescribed was $14.05 \%$ and with an injection prescribed was $46.01 \%$ and percentage of drugs prescribed from essential drug list was $64.4 \%$.

There was a significant difference in the total number of drugs, antihypertensive drugs, antimicrobial agents and other drugs used in patients with impaired renal function when compared to patients with normal renal function. A mean of $9.64 \pm 3.123$ drugs per patients were used in present study population and a mean of $10.05 \pm 3.081$ drugs per patient were used in patients with impaired renal function. Polypharmacy was seen in the present study and is a common finding in majority of studies in India in elderly and in patients with impaired renal function which further increases the risk of drug interactions and adverse drug reactions which are relatively common in elderly and in patients with impaired renal function.

Percentage of drugs prescribed in generic names was minimal when compared to drugs prescribed in brand names, which was in majority in the present study. Generic drug prescription would be advised since majority of the patients are from class IV socioeconomic status and generic drugs prescriptions are cost effective.

Appropriate drug selection for patients with CKD is important to avoid unwanted drug effects and to ensure optimal patient outcomes. Rational drug prescription is difficult in CKD patients due to a higher risk of drugrelated problems since they need complex therapeutic regimens requiring frequent monitoring and dosage adjustments. The presence of other comorbidities such as diabetes mellitus, hypertension, coronary artery disease, and infections make the situation more complicated. Inappropriate medication use can increase adverse drug effects as reflected in prolonged hospital stays, increased health care utilization and costs. ${ }^{23}$

The median (IQR) number of drugs per prescription was 10 (9-13) which indicates polypharmacy. Polypharmacy has been defined as the use of 5 or more medications to one patient at a time. ${ }^{24}$ However, in view of the complex nature and coexisting comorbidities of $\mathrm{CKD}$, some researchers suggest the use of more than 9 drugs at a time to be considered polypharmacy. ${ }^{24}$ Inappropriate polypharmacy can lead to significant morbidities and mortality. ${ }^{25}$

The accumulation and toxicity of many drugs can develop rapidly if dosages are not adjusted in patients with renal 
impairment. ${ }^{26}$ Kidney disease studies have shown that the cases of chronic kidney disease are growing dramatically over the past 20 years. $^{27,28}$

More than half of the adverse drug effects are due to the inappropriate dosage adjustments. ${ }^{29}$ Correctly adjusting the drug dosage in renal dysfunction, contributes to fewer adverse drug effects and decreases the therapeutic costs, hospitalization, length of hospital stays and mortality as well as maintaining therapeutic effectiveness. ${ }^{30,31}$

This study included only 165 prescriptions for analysis. Additionally, this was only a unicentric study. A largescale multicentric study would be the future option to conclusively analyse the prescription pattern.

\section{ACKNOWLEDGEMENTS}

Authors would like to thank Staff - Department of Pharmacology, SDM College of Medical Sciences and Hospital.

\section{Funding: No funding sources}

Conflict of interest: None declared

Ethical approval: The study was approved by the Institutional Ethics Committee

\section{REFERENCES}

1. Levey AS, Coresh J. Chronic kidney disease. The lancet. 2012 Jan 14;379(9811):165-80.

2. Levey AS, Coresh J, Bolton K, Culleton B, Harvey $\mathrm{KS}$, Ikizler TA, et al. K/DOQI clinical practice guidelines for chronic kidney disease: evaluation, classification, and stratification. Am J Kidney Dis. 2002;39(2 SUPPL. 1).

3. Manley HJ, Drayer DK, Muther RS. Medicationrelated problem type and appearance rate in ambulatory hemodialysis patients. BMC Nephrol. 2003 Dec;4(1):10.

4. Kappel J, Calissi P. Nephrology: 3. Safe drug prescribing for patients with renal insufficiency. Canad Med Assoc J. 2002 Feb 19;166(4):473-7.

5. World Health Organization: Burden of Disease Project. Available

at http://www3.who.int/whosis/menu.cfm (accessed on 2014 Nov).

6. Roberts JA, Lipman J. Pharmacokinetic issues for antibiotics in the critically ill patient. Cri Care Med. 2009 Mar 1;37(3):840-51.

7. Wanigasuriya KP, Peiris-John RJ, Wickremasinghe R, Hittarage A. Chronic renal failure in North Central Province of Sri Lanka: an environmentally induced disease. Transactions Royal Soc Trop Med Hygiene. 2007 Oct 1;101(10):1013-7.

8. Le Grand A, Hogerzeil HV, Haaijer-Ruskamp FM. Intervention research in rational use of drugs: a review. Health Policy Planning. 1999 Jan 1;14(2):89102.
9. Levey AS, Coresh J, Balk E, Kausz AT, Levin A, Steffes MW, et al. National Kidney Foundation practice guidelines for chronic kidney disease: Evaluation, classification, and stratification. Ann Intern Med. 2003;139:137-47.

10. Long CL, Raebel MA, Price DW, Magid DJ. Compliance with dosing guidelines in patients with chronic kidney disease. Ann Pharmacother. 2004 May;38(5):853-8.

11. Manley HJ, Cannella CA, Bailie GR, Peter WL. Medication-related problems in ambulatory hemodialysis patients: a pooled analysis. Am J Kidney Dis. 2005 Oct 1;46(4):669-80.

12. WHO Collaborating Centre for Drug Statistics Methodology (2009) Guidelines for ATC classification and DDD assignment. 2010. Accessed on 2013 Jan 05. Available at http://www.whocc.no/filearchive/publications/2010g uidelines.pdf.

13. Koristkova B, Grundmann M, Brozmanova $\mathrm{H}$. Differences between prescribed daily doses and defined daily doses of antiepileptics--therapeutic drug monitoring as a marker of the quality of the treatment. Int J Clin Pharmacol Therapeut. 2006 Sep 1;44(9).

14. Muller A, Monnet DL, Talon D, Hénon T, Bertrand X. Discrepancies between prescribed daily doses and WHO defined daily doses of antibacterials at a university hospital. Br J Clin Pharmacol. 2006 May;61(5):585-91.

15. Duarte-Ramos F, Cabrita J. Using a pharmacoepidemiological approach to estimate diabetes type 2 prevalence in Portugal. Pharmacoepidemiol Drug Safety. 2006 Apr;15(4):269-74.

16. Grimmsmann T, Himmel W. Discrepancies between prescribed and defined daily doses: a matter of patients or drug classes?. Eur J Clin Pharmacol. 2011 Aug $1 ; 67(8): 847-54$.

17. Ramachandran G, Rohith V, Topno I. Evaluation of prescribing pattern of anti-diabetic drugs using WHO prescribing indicators in a tertiary care hospital in Puducherry: a cross-sectional study. Pharma Innovation. 2015 Jul 1;4(5, Part B):76.

18. Santra S, Agrawal D, Kumar S, Mishra SS. A study on the drug utilization pattern in patients with chronic kidney disease with emphasis on antibiotics. J Integrative Nephrol Androl. 2015 Jul 1;2(3):85.

19. Abraham F, Varughese G, Mathew JC, John PM, Sam GK. Drug utilization pattern among geriatric patients in a tertiary care teaching hospital. Asian J Pharm Clin Res. 2015;8(6):191-4.

20. Bajait CS, Pimpalkhute SA, Sontakke SD, Jaiswal $\mathrm{KM}$, Dawri AV. Prescribing pattern of medicines in chronic kidney disease with emphasis on phosphate binders. Indian J Pharmacol. 2014 Jan;46(1):35.

21. Pavitra RY, Geeta M, Aggarwal R, Somashekhar HS. Drug utilization pattern of antihypertensive drugs in chronic kidney disease patients in a tertiary care hospital. J Dental Med Sci. 2014;13(11):23-7. 
22. Jhaveri BN, Patel TK, Barvaliya MJ, Tripathi CB. Drug utilization pattern and pharmacoeconomic analysis in geriatric medical in-patients of a tertiary care hospital of India. J Pharmacol Pharmacotherapeut. 2014 Jan;5(1):15.

23. Al-Ramahi R. Medication prescribing patterns among chronic kidney disease patients in a hospital in Malaysia. Saudi J Kidney Dis Transplantation. 2012 Mar 1;23(2):403.

24. Bajait CS, Pimpalkhute SA, Sontakke SD, Jaiswal KM, Dawri AV. Prescribing pattern of medicines in chronic kidney disease with emphasis on phosphate binders. Indian J Pharmacol. 2014 Jan;46(1):35.

25. Bushardt RL, Massey EB, Simpson TW, Ariail JC, Simpson KN. Polypharmacy: misleading, but manageable. Clin Interventions Aging. 2008 Jun;3(2):383.

26. Swan SK, Bennett WM. Drug dosing guidelines in patients with renal failure. Western J Med. 1992 Jun;156(6):633.

27. U.S Renal Data System (USRDS) Annual Data Report: atlas of end-stage renal disease in the United States. Bethesda: national institutes of health, national institute of diabetes and digestive and kidney diseases; 2006.
28. Coresh J, Selvin E, Stevens LA, Manzi J, Kusek JW, Eggers P, et al. Prevalence of chronic kidney disease in the United States. JAMA. 2007 Nov 7;298(17):2038-47.

29. van Dijk EA, Drabbe NR, Kruijtbosch M, De Smet PA. Drug dosage adjustments according to renal function at hospital discharge. Ann Pharmacother. 2006 Jul;40(7-8):1254-60.

30. Falconnier AD, Haefeli WE, Schoenenberger RA, Surber C, Martin-Facklam M. Drug dosage in patients with renal failure optimized by immediate concurrent feedback. J General Internal Med. 2001 Jun;16(6):369-75.

31. Liano F, Pascual J, Madrid Acute Renal Failure Study Group. Epidemiology of acute renal failure: a prospective, multicenter, community-based study. Kidney Int. 1996 Sep 1;50(3):811-8.

Cite this article as: Bhandari PR, Bhandary A. Drug usage pattern of antimicrobials in elderly hypertensive, diabetic in-patients with or without impaired renal function. Int J Basic Clin Pharmacol 2018;7:1946-53 